\title{
Mode mixing in Antiferromagnetically Correlated Double Quantum Wells
}

\author{
R. J. Radtke \\ Division of Engineering and Applied Sciences, \\ Harvard University, Cambridge, Massachusetts 02138 \\ S. Das Sarma \\ Condensed Matter Theory Group, Department of Physics, \\ University of Maryland, College Park, Maryland 20742-4111 \\ A. H. MacDonald \\ Department of Physics, Indiana University, Bloomington, Indiana 47405
}

(Received by Phys. Rev. B on 7 July 1997)

\begin{abstract}
We examine the robustness of a recently predicted exchange-induced zero-field magnetic phase in semiconductor double quantum wells in which each well is spin-polarized and the polarization vectors are antiparallel. Magnetic instabilities are a general feature of Coulombic double quantum well systems at low densities. We argue that this antiferromagnetic phase is stabilized relative to ferromagnetic ones by an effective superexchange interaction between the wells. Detailed self-consistent Hartree-Fock calculations using a point-contact model for the interaction show that the antiferromagnetic phase survives intra-subband repulsion matrix elements neglected in earlier work in a large portion of the model's parameter space. We also examine the role of asymmetry due to biasing or to differences in the widths of the two quantum wells. The asymmetry creates a mode coupling between the intra- and inter-subband collective spin-density excitations (SDEs) which changes the Raman spectroscopy signature of the phase transition from a complete softening of the inter-subband SDE to a cusp as the density is tuned through the transition. This cusp may be detectable in inelastic light scattering experiments in samples of sufficient quality at low enough temperatures and densities.

PACS numbers: 73.20.Dx, 73.20.Mf, 75.70.Cn, 71.10.-w
\end{abstract}

\section{INTRODUCTION}

The subject of exchange-correlation-induced phase transitions has proven to be a rich field of research which has revealed many intriguing phenomena. Normal Fermiliquid-state instabilities occur when the kinetic energy of the particles in a quantum system is of the same order or smaller than the inter-particle exchange and correlation energies. The instabilities lead to a variety of new electronic states. In Coulombic systems, this situation obtains at low densities and instabilities are more likely in systems of reduced dimensionality or, especially in two dimensions, in an applied magnetic field. Perhaps the best known examples of such new states occur in the in the fractional-quantum-Hall, strong-field regime of twodimensional electronic systems. 1

The interest in exchange-correlation-induced phase transitions can be traced in part to the availability of high-quality semiconductor quantum wells, quantum wires, and superlattices. These artificial structures may be fabricated with remarkable precision and quality and possess electron densities that can be varied over a large range through a combination of modulation doping and judicious gating. The one- or two-dimensional nature of the resulting electronic systems, and the low densities realizable in devices of this kind, make them ideal for studies of interaction-driven physics. Another feature of these devices of importance to the current work is the ex- tra degree of freedom available when multiple layers are present, as in multiple quantum wells or superlattices. This new degree of freedom allows transitions to states with order not only in the intra-layer electronic degrees of freedom but alspindiagonal or off diagonal inter-layer charge 13 or spin 101418 observables.

In particular, a great deal of attention has recently focussed on the possibility of inter-layer spin ordering in wide single or double quantum wells. This attention is motivated by studies of quantum well structures in which the lowest two subbands are well separated in energy from the higher subbands and the density is sufficiently low so that only these two subbands are occupied. In these structures, an earlier theoretical calculation of the collective spin-density excitations (SDEs) in the absence of a magnetic field showed a complete softening of the inter-subband SDE in a range of densities around that at which the second subband begins to populate.14 Subsequent analysis indicated that this softening corresponded to a phase transition to a state in which each well in the double quantum well (or the electron gases on each side of a wide single quantum well) was spin polarized with the polarization vectors antiparallel; i.e., a transition to an antiferromagnetic order in the well spin densities 15 Further work predicted that a similar transition to a canted antiferromagnetic phase should occur in the presence of a magnetic field at $\nu=2,17$ and this transition seems to have been observed experimentally. 16 However, the pre- 
dicted transition in the zero-field case has not yet been observed.18

Several possibilities exist which may explain the absence of the zero-field antiferromagnetic phase in these experiments. The calculations predicting this phase 15 are based on a mean-field treatment of the interacting system, which is known to overestimate the densities and temperatures at which such symmetry-breaking transitions occur. In addition, electronic scattering by disorder or impurities generally has a detrimental effect on correlation-induced phases. Both of these difficulties are exacerbated by the low dimensionality of the double quantum well system considered. Thus, the zerofield antiferromagnetic phase may exist but may not have been observed due to measurements made at temperatures which are too large in samples of insufficiently high quality. The fact that the $\nu=2$ transition is bserved 16 as predicted by the mean-field calculations 17 does not contradict this point of view, since the magnetic field completely quenches the kinetic energy and makes the mean-field theory a controlled approximation to the interacting system.

Alternative explanations for the absence of the zerpfield phase lie in the structure of the theory itself, 15 which was derived to explore the qualitative features of the antiferromagnetic phase without considering several confounding effects which may nonetheless be important. In particular, the previous calculation 15 did not account for interactions between electrons in the same subband, which should be of the same size or stronger than the interactions between electrons in different subbands which were included. This omission can become especially important when the intersubband excitation softens. These interactions could introduce ferromagnetic or charge-ordered phases into the model which are not probed by the current experiments. More seriously, the slight asymmetry present in any realistic double quantum well structure will couple the intra- and inter-subband SDEs, potentially preventing the latter from softening. As this softening was expected to be hallmark of the antiferromagnetic phase transition, 14,15 it seemed reasonable to hypothesize that any asymmetry in the structure might suppress the antiferromagnetic phase entirely.

In this paper, we address the question of the robustness of the zero-field antiferromagnetic phase in the presence of intra-subband interactions and asymmetry in the double quantum well structure. First, we argue on general grounds that the antiferromagnetic phase is a direct consequence of the importance of the intra-well exchange interaction at low densities and is stabilized by the interwell hopping, which leads to an effective superexchange interaction. Thus, this phase should obtain in a suitably constructed heterostructure. Second, we extend the selfconsistent Hartree-Fock calculations of earlier work 15 to include both intra- and inter-subband matrix elements of the model interaction and the effects of an asymmetric double quantum well. These calculations demonstrate that, while the intra-subband interaction does introduce ferromagnetic phases and asymmetry does reduce the region of the phase diagram occupied by the antiferromagnetic phase, the antiferromagnetic phase does not disappear. By examining the collective mode spectrum in the asymmetric structure, we also find that the intersubband SDE does not soften due to the mode coupling between the intra- and inter-subband SDEs. The antiferromagnetic transition nevertheless occurs as a result of the collapse of the intra-subband SDE which, through the mode-coupling, has a strongly antiferromagnetic character.

The outline of this paper is as follows. In the next Section, we employ a simple model for two weakly coupled two-dimensional electron gases to examine the energetics of the antiferromagnetic transition. Sec. III contains the formalism for the extended self-consistent Hartree-Fock theory used in the remainder of the paper. This formalism includes asymmetry and all matrix elements of the interaction and is used to compute both the ground state and collective mode properties in what follows. In Sec. IV, we present the results of our computations for the matrix elements, phase diagram, and collective modes in this model and discuss their implications. Finally, in Sec. V, we summarize our results and conclude.

\section{ORIGIN AND STABILITY OF THE ANTIFERROMAGNETIC PHASE}

In this Section, we examine a simple model of a double quantum well in order to extract the basic physics underlying the zero-field antiferromagnetic phase. To that end, consider two two-dimensional electron gases separated by a barrier constructed so that the interaction between them is negligible. This would be the case if the two-dimensional layers were widely separated or the barrier were very high and if the dielectric constant of the barrier were large. Suppose the electrons in each layer move freely except for a Hubbard-like point-contact interaction $V(\mathbf{r})=V_{0} \delta(\mathbf{r})$ as used in textbook treatments of itinerant magnetism. 19

For equal charge density in the two layers, the HartreeFock energy $E_{\mathrm{HF}}$ of the two-layer system can be derived following Refs. 2, 3,10 and may be expressed in the form

$$
\frac{E_{\mathrm{HF}}}{A}=\frac{n^{2}}{8 N_{0}}\left(1-N_{0} V_{0}\right)\left(\frac{1+m_{1}^{2}}{2}+\frac{1+m_{2}^{2}}{2}\right)+\frac{1}{4} V_{0} n^{2},
$$

where $n$ is the total electronic density in both layers, $N_{0}$ is the single-spin, two-dimensional density of states, and

$$
m_{i}=\frac{n_{i \uparrow}-n_{i \downarrow}}{n_{i \uparrow}+n_{i \downarrow}}
$$

is the relative spin polarization in layer $i=1,2$ with partial spin-dependent densities $n_{i \sigma}$. In this equation, the first term represents the contribution to the energy 
from combined kinetic and exchange effects, and the last term is the Hartree contribution. As is clear from Eq. (1), when $N_{0} V_{0}>1$, it is energetically favorable for both layers to acquire a spontaneous spin polarization $\left|m_{1}\right|=\left|m_{2}\right|=1$, while for $N_{0} V_{0}<1$, the layer remain unpolarized. This result is simply the Stoner criterion for $2 \mathrm{D}$ itinerant magnetism. 19

This description of spontaneous spin-polarization in two-dimensional electron systems is unrealistic both in the use of a point-contact interaction and in the use of the Hartree-Fock approximation. Our objective in this section is to obtain a qualitative understanding of the influence of weak electronic tunneling between the layers on a double-layer system when isolated single layer systems are close to their ferromagnetic instabilities. We postpone a realistic discussion of the system parameters for which the physics we address in this paper is likely to realized to Sec. V. For a sufficiently strong intra-layer repulsion, then, the exchange interaction forces both layers to spin polarize, but the relative orientation of the polarizations is unknown. For simplicity, let us restrict our attention to two possibilities for the relative orientation: parallel (ferromagnetic) alignment or antiparallel (antiferromagnetic) alignment. With the spin unpolarized (paramagnetic) phase, the three possible phases for the two-layer system are shown in Fig. 11.

In the absence of any inter-layer coupling, the ferromagnetic and antiferromagnetic phases are degenerate, but this situation changes if we introduce a small amplitude for hopping between the layers. Writing $c_{i \mathbf{k} \sigma}$ for the annihilation operator of an electron in layer $i=1,2$ with two-dimensional wave vector $\mathbf{k}$ and spin projection $\sigma$, this inter-layer hopping is governed by the Hamiltonian

$$
H_{\perp}=-\frac{\Delta_{0}}{2} \sum_{\mathbf{k} \sigma}\left[c_{1 \mathbf{k} \sigma}^{\dagger} c_{2 \mathbf{k} \sigma}+\text { h.c. }\right]
$$

where $\Delta_{0}$ is the splitting between the symmetric and antisymmetric single-particle eigenstates. The leading order change in the ground state energy due to $H_{\perp}$ can be calculated using linear response theory, and is proportional to the transverse pseudospin susceptibility of double-layer systems defined in Ref. 20. For the present model with no inter-layer interactions and contact intralayer interactions, the time-dependent Hartree-Fock approximation for the susceptibility gives

$$
\frac{\delta E}{A}= \begin{cases}-\frac{1}{2} N_{0} \Delta_{0}^{2} & \text { (paramagnetic) } \\ -\frac{1}{4} N_{0} \Delta_{0}^{2} & \text { (ferromagnetic) } \\ -\frac{1}{2} \Delta_{0}^{2} / V_{0} & \text { (antiferromagnetic) }\end{cases}
$$

The fact that the results for paramagnetic and ferromagnetic states are independent of the interaction strength is a special property of the present model related to both the absence of interlayer interactions and the wave vector independence of the exchange self-energy.

We see that, in all cases, introducing inter-layer hopping reduces the ground state energy, as one might expect when the confinement of the electrons to the layers

\section{Paramagnetic \\ Phase}
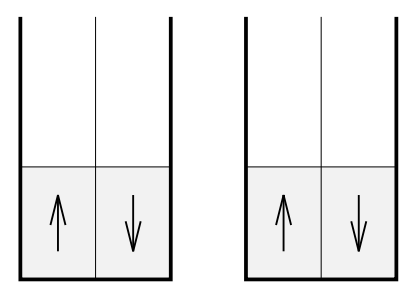

\section{Ferromagnetic \\ Phase}

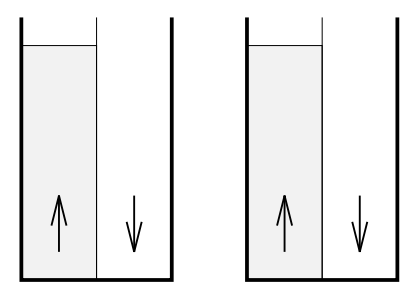

\section{Antiferromagnetic \\ Phase \\ Left \\ Well \\ Right \\ Well}

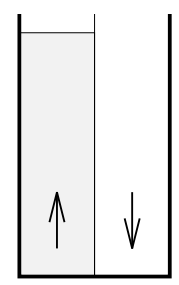

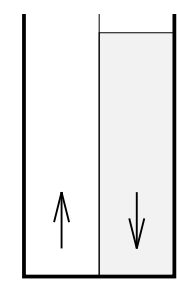

FIG. 1. Population of spin and well states for the phases of a double quantum well considered in Sec. III. In the paramagnetic phase, there is no spin polarization in either well. In the magnetic phases, each well is completely spin-polarized with the polarization vectors parallel in the ferromagnetic phase and antiparallel in the antiferromagnetic phase. 
is weakened. Comparing the energies of the ferromagnetic and antiferromagnetic phases, we also observe that the degeneracy between these phases is broken by the hopping term. Specifically, the antiferromagnetic phase is found to be more stable than the ferromagnetic phase if $N_{0} V_{0}<2$, implying that the inter-layer hopping opens up a region of antiferromagnetic order between the paramagnetic and ferromagnetic phases. The mechanism for this stabilization can be deduced from the form of $\delta E / A$ to be a superexchange interaction; that is, an electron is able to hop from one layer to the other and back at the cost of a Hubbard energy in the intermediate state, yielding an energy savings of $\Delta_{0}^{2} / 2 V_{0}$. A similar mechanism is blocked by the Pauli exclusion principle in the ferromagnetic phase, since the hopping Hamiltonian preserves spin [Eq. (3)].

We argue that this mechanism favoring an antiferromagnetic arrangement of the ordered moments in the two layers will be dominant in most circumstances. The calculations described below indicate that an effective superexchange interaction between the wells should stabilize an antiferromagnetic phase for moderate interaction strengths. This explanation of the zero-field antiferromagnetic phase suggests why the presence of asymmetry and additional interaction matrix elements may not eliminate this phase: both the two-dimensional ferromagnetism within each well and the superexchange interaction between the wells should be fairly insensitive to these perturbations. In the remainder of the paper, we perform a more detailed self-consistent Hartree-Fock calculation to support this statement and to explore the consequences of these perturbations.

\section{FORMALISM}

To accomplish the goal of investigating matrix element and asymmetry effects, we employ an extension of the point-contact model described in Ref. 15. In the original calculation, the full Coulomb interaction between the electrons in the double quantum well was approximated by a delta function in real space with only the matrix elements of this interaction between the lowest two subbands being kept and the remainder being set to zero. 15 The use of a delta-function or point-contact interaction is based primarily on a desire to create a simple, solvable model which mimics the qualitative features of the fully interacting system. A quantitative theory would require that the interaction be made realistic, and also that the interactions be treated more accurately than in the Hartree-Fock approximation. In practise this would require quantum Monte-Carlo calculations of some type, which would involve an enormous amount of effort and would not be able to address the excitation spectrum which provides the experimental signature for the state we are proposing. We therefore maintain the pointcontact form of the interaction here. However, there is

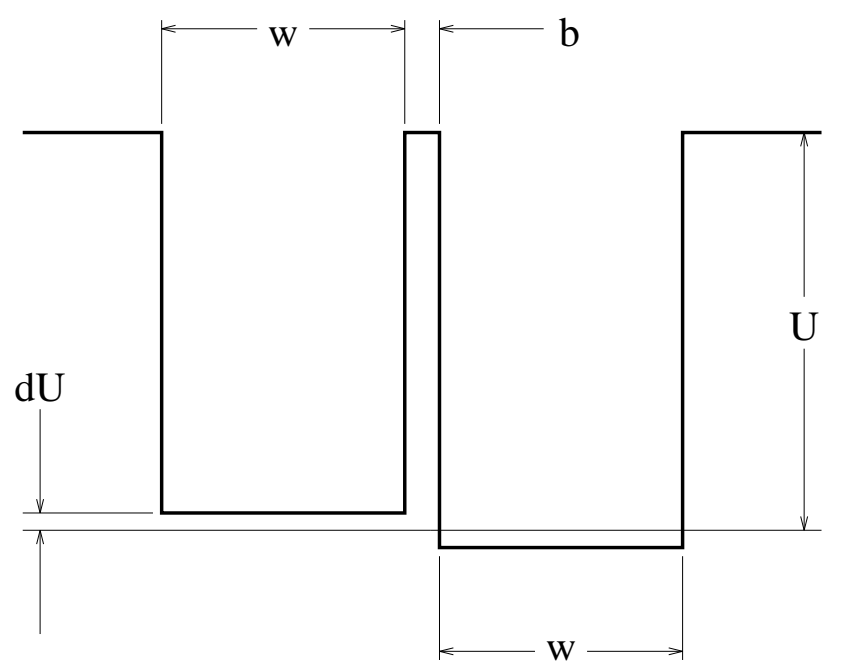

FIG. 2. Diagram of the asymmetric double quantum well considered in this paper indicating the well width $w$, barrier width $b$, confining potential $U$, and potential asymmetry $d U$. The values of the parameters used here and in previous studies of magnetic instabilities of these system, 14,5 model a typical $\mathrm{GaAs} / \mathrm{Al}_{x} \mathrm{Ga}_{1-x} \mathrm{As}$ double quantum well with $w=140 \AA$, $b=30 \AA$, and $U=220 \mathrm{meV}$. We consider both symmetric $(d U=0)$ and weakly asymmetric $(d U=0.5 \mathrm{meV})$ double quantum wells in what follows.

no reason to set matrix elements of this interaction other than those between the lowest subbands to zero, as in previous work. Indeed, we shall see below that these other matrix elements are of the same order as the intersubband ones. Thus, we shall include all the matrix elements between the lowest two subbands in our calculations.

In addition to the issue of intra-subband repulsion, we would also like to study the effects of quantum well asymmetry on the phase diagram and collective modes of this system. This asymmetry arises in real quantum wells through alloy fluctuations across the profile of the well or fluctuations in the well thickness, and we model it by allowing one of the wells to be deeper than the other, as illustrated in Fig. 2. Although we have assumed the effective asymmetry to enter through the well depth, the results of our calculations for systems with asymmetric well widths should be qualitatively similar.

We therefore consider a three-dimensional electron gas confined along the $z$-direction by a potential $V_{C O N F}(z)$ of the type shown in Fig. 2 which interacts through a three dimensional point-contact potential $V(\mathbf{R})=V \delta(\mathbf{R})$. In the absence of the interaction $V(\mathbf{R})$, the electronic eigenstates are given by the solution of the time-independent Schrödinger equation

$$
\left[-\frac{\hbar^{2}}{2 m^{*}} \frac{d^{2}}{d z^{2}}+V_{C O N F}(z)\right] \xi_{n}(z)=\epsilon_{n} \xi_{n}(z)
$$

where $m^{*}$ is the effective mass, which is assumed to be constant throughout the heterostructure. Normalizing these eigenfunctions by 


$$
\int d z\left|\xi_{n}(z)\right|^{2}=1
$$

we write the electron annihilation operator $\psi_{\sigma}(\mathbf{R})$ as

$$
\psi_{\sigma}(\mathbf{R})=\frac{1}{\sqrt{A}} \sum_{n \mathbf{k}} e^{i \mathbf{k} \cdot \mathbf{r}} \xi_{n}(z) c_{n \mathbf{k} \sigma}
$$

where $\mathbf{R}=(\mathbf{r}, z)=(x, y, z), \mathbf{k}=\left(k_{x}, k_{y}\right), A$ is the transverse area of the sample, and $c_{n \mathbf{k} \sigma}$ annihilates a quasiparticle in subband $n$, of transverse wave vector $\mathbf{k}$, and with spin projection $\sigma$ (these conventions will be used throughout this paper).

Defining a composite subband and spin index $a=$ $\left(n_{a}, \sigma_{a}\right)$ with summation over repeated indices implied, the Hamiltonian for this system is

$$
\begin{aligned}
H= & \sum_{\mathbf{k}} \epsilon_{a \mathbf{k}} c_{a \mathbf{k}}^{\dagger} c_{a \mathbf{k}} \\
& +\frac{1}{2 A} \sum_{\mathbf{k k}^{\prime} \mathbf{q}} V_{a d, b c} c_{a \mathbf{k}+\mathbf{q}}^{\dagger} c_{b \mathbf{k}^{\prime}-\mathbf{q}}^{\dagger} c_{c \mathbf{k}^{\prime}} c_{d \mathbf{k}} .
\end{aligned}
$$

Here, the quasiparticle energy

$$
\epsilon_{a \mathbf{k}}=\epsilon_{n}+\frac{\hbar^{2} k^{2}}{2 m^{*}}-\mu
$$

is measured with respect to the chemical potential $\mu$ and the matrix elements of the interaction are

$V_{a b, c d}=\delta_{\sigma_{a} \sigma_{b}} \delta_{\sigma_{c} \sigma_{d}} V \int d z \xi_{n_{a}}^{*}(z) \xi_{n_{b}}(z) \xi_{n_{c}}^{*}(z) \xi_{n_{d}}(z)$.

We treat this Hamiltonian within self-consistent Hartree-Fock theory allowing for the possibility of phases with broken symmetry in subband and spin indices, but imposing translational invariance within each layer. The electronic Green's function in the interacting system can therefore be written

$$
G_{a b}\left(k_{n}\right)=-\int_{0}^{\beta} d \tau e^{i \omega_{n} \tau}\left\langle T_{\tau}\left[c_{a \mathbf{k}}(\tau) c_{b \mathbf{k}}^{\dagger}(0)\right]\right\rangle,
$$

where $k_{n}=\left(\mathbf{k}, i \omega_{n}\right), \beta=1 / T\left(\hbar=k_{B}=1\right.$ throughout this paper), and the rest of the notation is standard.21 This Green's function is determined self-consistently from the self-energy in the Hartree-Fock approximation,

$$
\Sigma_{a b}=\left[V_{a b, d c}-V_{a c, d b}\right] \frac{T}{A} \sum_{k_{m}^{\prime}} e^{-i \omega_{m} 0-} G_{c d}\left(k_{m}^{\prime}\right),
$$

and the Dyson equation,

$$
\left[\left(i \omega_{n}-\epsilon_{a \mathbf{k}}\right) \delta_{a b}-\Sigma_{a b}\right] G_{b c}\left(k_{n}\right)=\delta_{a c},
$$

under the constraint of constant sheet density $N_{s}$,

$$
N_{s}=\frac{T}{A} \sum_{k_{m}} e^{-i \omega_{m} 0-} G_{a a}\left(k_{m}\right),
$$

which determines the chemical potential.

We solve these equations in the following way. Given a double quantum well structure defined by the effective mass $m^{*}$, sheet density $N_{s}$, and the structural parameters shown in Fig. 2, we compute the eigenfunctions and eigenenergies by solving Eq. (5) with the normalization condition Eq. (6). These eigenfunctions are used to compute the matrix elements of the interaction via Eq. (10) in terms of a single parameter $\mathrm{V}$. The resulting matrix elements and eigenenergies are employed to solve Eqs. (11)(14) self-consistently at $T=0$ following the procedure outlined in Sec. II.B of Ref. 15 and including all matrix elements of the interaction between the lowest two subbands. This procedure yields the ground state properties of the system as a function of the sheet density $N_{s}$, the interaction parameter $V$, and the structural parameters of the double quantum well [Fig. 2].

To illuminate the properties of the interacting system further, we also compute the generalized density-density response function, which is defined by the analytic continuation of

$$
\begin{aligned}
& \Pi^{\mu}\left(\mathbf{Q}, i \nu_{n}\right)=-\int \frac{d \mathbf{R}}{\mathcal{V}} e^{-i \mathbf{Q} \cdot \mathbf{R}} \int_{0}^{\beta} d \tau e^{i \nu_{n} \tau} \times \\
& \left\langle T_{\tau}\left[\rho^{\mu}(\mathbf{R}, \tau) \rho^{\mu}(\mathbf{0}, 0)\right]\right\rangle
\end{aligned}
$$

to real frequencies. In this expression, $\mathcal{V}$ is the system volume and the generalized density operator is

$$
\rho^{\mu}(\mathbf{R})=\sum_{\sigma \sigma^{\prime}} \psi_{\sigma}^{\dagger}(\mathbf{R}) \sigma_{\sigma \sigma^{\prime}}^{\mu} \psi_{\sigma^{\prime}}(\mathbf{R})
$$

with $\psi_{\sigma}(\mathbf{R})$ given by Eq. (7) and $\sigma^{\mu}=\left(1, \sigma^{x}, \sigma^{y}, \sigma^{z}\right)$ are the Pauli matrices. This response function is computed from the non-interacting response function in subband and spin space within the conserving approximation described in Sec. V.A of Ref. 15 but with the inclusion of all interaction matrix elements. In addition to the information this response function reveals about the excitations of the interacting system, its imaginary part is proportional to the intensity observed in resonant inelastic light scattering measurements, 223 allowing us to make contact with experiment. This is particularly relevant here, because searches for the antiferromagnetic phase in both finite 16 and zero 18 magnetic field have employed this technique.

\section{RESULTS}

In this Section, we apply the formalism described in Sec. III to compute the ground state phase diagram and collective modes in a typical $\mathrm{GaAs} / \mathrm{Al}_{x} \mathrm{Ga}_{1-x} \mathrm{As}$ double quantum well structure which is expected to exhibit the zero-field antiferromagnetic instability. The structure has a well width of $140 \AA$, a barrier width of $30 \AA$, and a well depth of $220 \mathrm{meV}$ [cf. Fig. 2], and an electronic effective mass $m^{*}=0.067 m_{e}$. For the moment, we leave the asymmetry unspecified. 


\section{A. Matrix Elements}

As a first step in obtaining the phase diagram for this structure, we must solve the time-independent Schrödinger equaution [Eq. (5)] for the lowest two eigenfunctions $\xi_{n}(z)$ and eigenenergies $\epsilon_{n}$ at a fixed value of the asymmetry parameter $d U$ [cf. Fig. 2] and then compute the matrix elements of the interaction through Eq. (10). Solving Eq. (5) is straightforward and yields a splitting of the non-interacting eigenstates of $\Delta_{\text {SAS }}^{0}=$ $\epsilon_{2}-\epsilon_{1}=2.25 \mathrm{meV}$ for $d U=0$. As $d U$ is increased, this splitting increases to a maximum of $18.5 \mathrm{meV}$ at $d U=9.4 \mathrm{meV}$; for larger $d U$, the lowest eigenfunction is localized in one well. Since the structures examined experimeptally have subband splittings on the order of $1 \mathrm{meV} 16.18$ we restrict our attention to small $d U$ values.

The dependence of the matrix elements on the asymmetry parameter is somewhat more interesting and merits a brief discussion. Since our model interaction is a delta function in real space and we have chosen the wave functions to be real, the matrix elements defined by Eq. (10) are invariant under permutation of the indices. Thus, there are only five independent matrix elements, $V_{11,11}, V_{22,22}, V_{11,22}, V_{11,12}$, and $V_{22,21}$, which are displayed in Fig. 3 as a function of the asymmetry parameter $d U$. In a symmetric double quantum well $(d U=0)$, the $V_{11,12}$ and $V_{22,21}$ matrix elements vanish by symmetry, but the remaining inter-subband $\left(V_{11,22}\right)$ and intrasubband $\left(V_{11,11}\right.$ and $\left.V_{22,22}\right)$ matrix elements are equal to within $5 \%$. We remark that for more realistic interaction models $V_{11,22}$, which is roughly 24 proportional to the difference of intra-layer and inter-layer interactions, is weaker than $V_{11,11}$ and $V_{22,22}$ which are roughly proportional to the sum. The latter matrix elements would be equal if the electrons were localized to the wells; the fact that they are nearly so indicates that the wave function overlap between the wells is small. Additionally, this calculation provides direct evidence that the neglect of the intra-subband repulsion employed in earlier work 15 is not generally justified for these double quantum well structures. We shall see, however, that their inclusion in the calculation changes the qualitative picture only slightly.

As the asymmetry is increased from zero, we discern several features. We see that the intra-subband matrix elements $V_{11,11}$ and $V_{22,22}$ are approximately equal and increase with increasing $d U$ to saturate at a value about twice the $d U=0$ one. The approximate equality of these diagonal matrix elements follows from the normalization condition, Eq. (6), imposed on the two eigenfunctions. The increase in these matrix elements with $d U$, on the other hand, can be attributed to the increasing confinement of the wave functions of the two subbands to opposite wells, similar to yhat occurs in the presence of an applied electric field 6 , Thus, at large $d U$, the two subband wave functions are almost completely localized in opposite wells, enhancing the magnitude of the diagonal matrix elements. For the same reason, the inter-

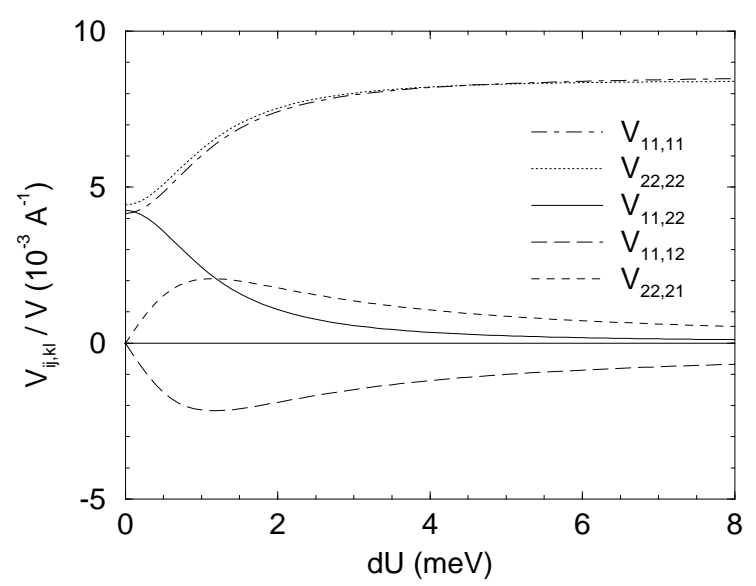

FIG. 3. Dependence of the interaction matrix elements $V_{i j, k l} / V$ [Eq. (10)] on the double quantum well asymmetry parameter $d U$ [cf. Fig. 2]. Shown are the intra-subband matrix elements $V_{11,11}$ (dot-dashed line) and $V_{22,22}$ (dotted line), the inter-subband matrix element $V_{11,22}$ (solid line), and the asymmetry-induced matrix elements $V_{11,12}$ (long dashed line) and $V_{22,21}$ (short-dashed line). For the point-contact interaction employed in this paper, the matrix elements are invariant under permutation of the indices, so these matrix elements span the entire set.

subband matrix element $V_{11,22}$ decreases with increasing asymmetry: as the wave functions from different subbands are increasingly localized, their overlap, and hence $V_{11,22}$, decreases to zero. Since the zero-field antiferromagnetic transition depends on this matrix element, it is clear that large asymmetry is inimical to this phase. Based on the small values of the observed splitting of the lowest two subbands in the experimental samples, 16,18 however, we expect the actual samples to be in a regime in which $V_{11,22}$ is still non-negligible. Hence, the antiferromagnetic phase is not immediately excluded.

Finally, we note that the mixing terms $V_{11,12}$ and $V_{22,21}$ have opposite signs and increase in magnitude with $d U$ to a maximum around $d U=1 \mathrm{meV}$. The wave function for the lowest $(n=1)$ subband has no nodes and we chose it to be positive. Orthogonality requires that the $n=2$ wave function have a node and we choose it to be negative in the well where $\left|\xi_{1}(z)\right|^{2}$ is largest; with this convention $V_{11,12}$ is negative and $V_{22,21}$ is positive, as observed in Fig. 3. In addition, since these matrix elements must vanish both in the symmetric $(d U=0)$ limit and when $d U$ is large and the subband wave functions are localized in different wells, the maximum seen in this figure is also expected. These results suggest that the mode coupling between intra- and inter-subband excitations induced by these matrix elements will be maximal around $d U=1 \mathrm{meV}$.

Taken together, the behavior of the interaction matrix elements presented in Fig. 3 indicate that the zerofield antiferromagnetic phase will probably not be stable against large asymmetry in the quantum wells. However, 
the current experimental samples have subband splittings more consistent with small asymmetry, and therefore these samples may be of high enough quality to observe this phase, at least in principle. To examine this situation further, we shall compute the phase diagram and collective modes for two choices of $d U$ in the weak asymmetry regime: $d U=0$ (the symmetric case) and $d U=0.5 \mathrm{meV}$ (the asymmetric case). The following subsections discuss the results of these calculations.

\section{B. Phase Diagram}

As described in the preceding subsection, the structure of the double quantum well yields the eigenenergies and eigenfunctions of the non-interacting system which are then used to compute the interaction matrix elements up to an overall factor $V$ [Eq. (10)]. To be consistent with earlier work, 15 we choose to parameterize the interaction strength by the magnitude of the inter-subband repulsion $V_{11,22}=V_{12}$ rather than by $V$, but all the matrix elements are uniquely determined by either parameter. With the structure and interaction strength fixed, the only other parameter in our model is the sheet density $N_{s}$. Given these parameters, Eqs. (11)-(14) can be solved at $T=0$ to yield the interacting ground state of the system. The resulting phase diagrams in terms of the dimensionless interaction strength $N_{0} V_{12}$ and sheet density $N_{s} / 2 N_{0} \Delta_{\text {SAS }}^{0}$ are presented in Fig. 专 for the double quantum well structure of Fig. 2. In these figures, $N_{0}$ is the single-spin, two-dimensional density of states, and $\Delta_{\mathrm{SAS}}^{0}=\epsilon_{2}-\epsilon_{1}$ is the subband splitting in the noninteracting system.

Consider the symmetric $(d U=0)$ case first [Fig. [- $1(\mathrm{a})]$. At first glance, this phase diagram is very similar to the one pbtained from the neglect of intra-subband repulsion 15 even though this repulsion is included in our calculations. At low density, the paramagnetic phase with one spin-degenerate subband occupied (labeled $N_{1}$ in the figure) is stable, while at higher densities $\left(N_{s} / 2 N_{0} \Delta_{\text {SAS }}^{0}>1\right)$ but weak interaction $\left(N_{0} V_{12}<1 / 2\right)$ the paramagnetic phase with two spin-degenerate subbands occupied (labeled $N_{2}$ ) is stable. For larger interaction strengths and intermediate densities, we see a broad region in which the antiferromagnetic $(A F)$ phase obtains. Thus, the inclusion of intra-subband repulsion does not eliminate the zero-field antiferromagnetic phase from the phase diagram of the model, despite the fact that the intra- and inter-subband repulsions are of the same order [Fig. 3].

These matrix elements do have an effect on the phase diagram, however. At higher densities $\left(N_{s} / 2 N_{0} \Delta_{\text {SAS }}^{0}\right.$ larger than approximately 2) and strong interaction $\left(N_{0} V_{12}>1 / 2\right)$, two different ferromagnetic phases appear. In these phases, each quantum well is spin polarized, and the polarization vectors are parallel. They differ in the arrangement and filling of the interacting
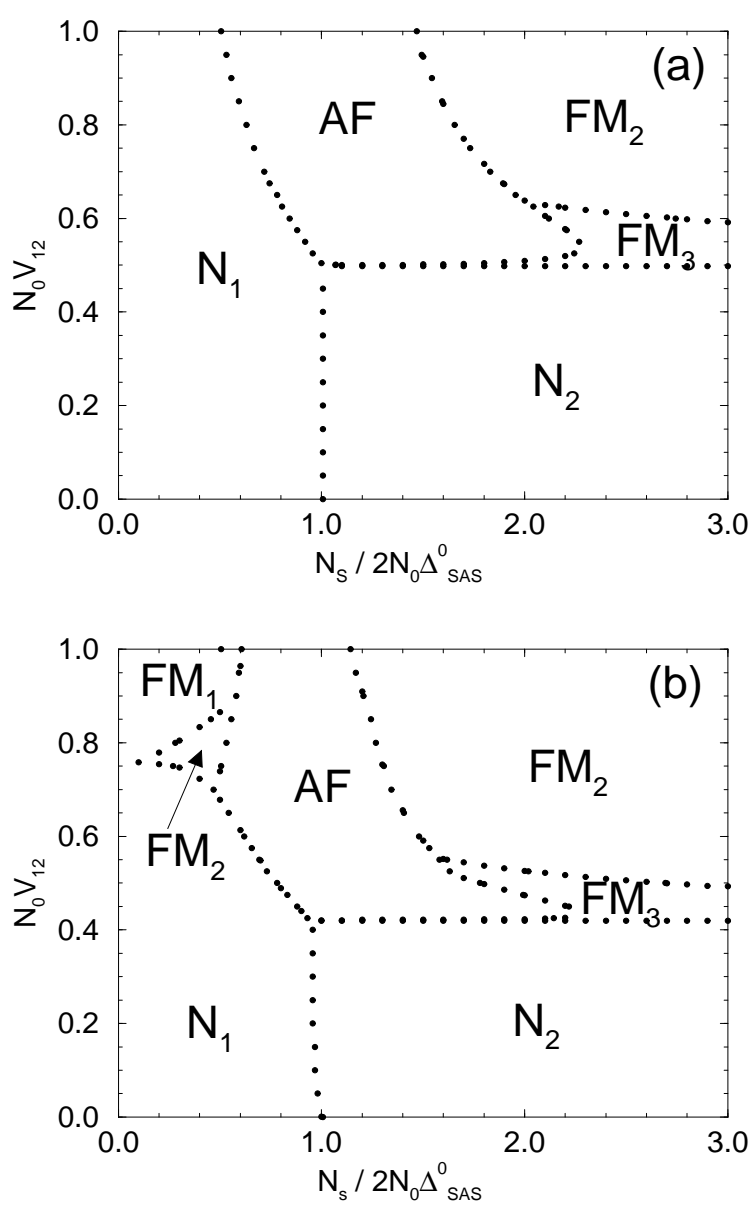

FIG. 4. Phase diagram of (a) a symmetric and (b) an asymmetric double quantum well in terms of the sheet density $N_{s}$ and the interaction parameter $V_{12}$ for the point-contact model described in Sec. III. The points are the numerically computed boundaries between the various phases of this model which are labeled as follows: $N_{1}$ and $N_{2}$ are paramagnetic phases with one and two spin-degenerate subbands occupied, respectively; $F M_{i}$ are ferromagnetic phases with $i$ non-degenerate subbands occupied; and $A F$ is the antiferromagnetic phase. See Fig. 1 for a real-space depiction of these phases and Fig. 2 for the structural parameters of the double quantum well. In the figure, $N_{0}$ is the single-spin, $2 \mathrm{D}$ electronic density of states, and $\Delta_{\text {SAS }}^{0}$ is the splitting between the lowest two states in the non-interacting system. Note that asymmetry reduces the extent of the antiferromagnetic phase in (b) but does not destroy it altogether. 
bands, as indicated by the labels in the figure: the phase $F M_{i}$ corresponds to an interacting band structure with $i$ spin-split subbands occupied. The presence of the ferromagnetic phases is expected when intra-subband repulsion in present; what is surprising is that the ferromagnetic phases do not exclude the presence of the antiferromagnetic phase. As argued in Sec. II based on a weakcoupling model, the antiferromagnetic phase is stabilized by a superexchange interaction for intermediate interaction strengths. Similar behavior is seen in Fig. \&(a) based on our strong-coupling computations and presumably originates from the same mechanism. Note that, in the limit of vanishing hopping between the wells, $V_{12}$ may be associated with $V_{0} / 2$ in the model of Sec. II, implying that the phase boundary $N_{0} V_{12}=1 / 2$ in Fig. 4 (a) is nothing but the Stoner criterion for the related Hubbard model. The fact that the model of Sec. II does not have a closer correspondence to Fig. 4(a) suggests that the relative magnitudes of the intra- and inter-subband interaction matrix elements, which are all equal in the model of Sec. II, are important for determining whether ferro- or antiferromagnetic phases obtain in a specific region of the phase diagram.

With the introduction of weak asymmetry $(d U=$ $0.5 \mathrm{meV}$ ), the qualitative features of the phase diagram do not change, as seen in Fig. @(b). As before, we find paramagnetic phases at small interaction strengths, the antiferromagnetic phase at larger interaction strengths and intermediate densities, and ferromagnetic phases at larger interaction strengths and higher densities. The identification of these phases precisely matches those in the symmetric case of Fig. A(a), although the position of the phase boundaries have shifted somewhat. An apparently new feature occurs at low density and large interaction strength, where an $F M_{1}$ ferromagnetic phase has replaced the paramagnetic $N_{1}$ phase. However, this phase also occurs in the symmetric structure when $N_{0} V_{12}>1$, but is cut-off in Fig. $4(\mathrm{a})$. It corresponds to the usual ferromagnetic instability in a single, spin-degenerate bandwhich obtains when the interaction is sufficiently strong.19

Although weak asymmetry clearly does not destroy the antiferromagnetic phase, it does have observable consequences. The most noticeable effect of the asymmetry on the ground state is that the spin polarizations in the magnetic phases, which can be obtained from the expectation value of the density operator, Eq. (16), are no longer of equal magnitude in the wells. This is an obvious consequence of an asymmetric structure which nonetheless does not disturb the identification of ferro- and antiferromagnetic phases, since one can determine whether the spin polarizations are parallel or antiparallel without referring to their magnitudes.

\section{Collective Modes}

The effects of asymmetry on the antiferromagnetic phase cannot be fully appreciated based on the groundstate properties alone, but must be augmented by an examination of the excitation spectrum of the system. We focus on the spin-density excitations in what follows, since they are the excitations most strongly coupled to the magnetic nature of the ground state and also be probed experimentally by Raman scattering.22 23 For these calculations, we compute the spin-spin response function $(\mu=3$ in Eq. (15)) for the appropriate ground state as discussed in Sec. III and identify the collective modes by peaks in the imaginary part of this response function. Since this procedure is used in the Raman scattering measurements, our results have direct implications for experiment and we shall discuss them in this context. For concreteness, we fix the interaction strength and sweep the sheet density through the second-order transition from the $N_{1}$ to the $A F$ phase in these calculations [cf. Fig. 价.

As an introduction to the general phenomenology of spin-density excitations in double quantum wells, consider the symmetric $(d U=0)$ case first. By appropriately arranging the light scattering geometry, Raman scattering can selecfizely probe the intersubband spin-density excitations, 1618 which, in our approximation, have the form shown in Fig. 5 (a). In addition to a continuum of inter-subband particle-hole excitations, there is a collective spin-density excitation (SDE) with a finite energy at $q=0$ which disperses with increasing $q$ toward the particle-hole continuum. The magnitude of the $q=0$ SDE energy is reduced from the subband splitting $\Delta_{\text {SAS }}$ by vertex corrections appearing in the response function due the the exchange interaction.25.26 In addition, there is an intra-subband SDE which has a linear-in-q dispersion in our model, shown by the dashed line in Fig. F)(a). If the well is symmetric, this mode will not appear in Raman spectra taken in a scattering geometry meant to observe inter-subband excitations. A symmetric system with identical quantum wells is invariant under inversion about the mid-point between the wells so that all states can be classified by a parity quantum number. Intersubband excitations, which are odd, and intrasubband excitations, which are even, do not interact and can cross as seen in Fig. 5(a).

As the density increases in our model, the exchangeinduced reduction in the $q=0$ inter-subband SDE energy increases until the mode softens entirely, as illustrated in Fig. 5(b). This complete softening was seen initially in time-dependent, local density approximation calculations of the SDE spectrum in these systems and was the first evidence for the zero-field antiferromagnetic phase. 14 An analysis of the real-space spin response identified this softening as an antiferromagnetic transition of the well spin polarizations. 15 As the density is increased past the antiferromagnetic transition, the inter-subband SDE 

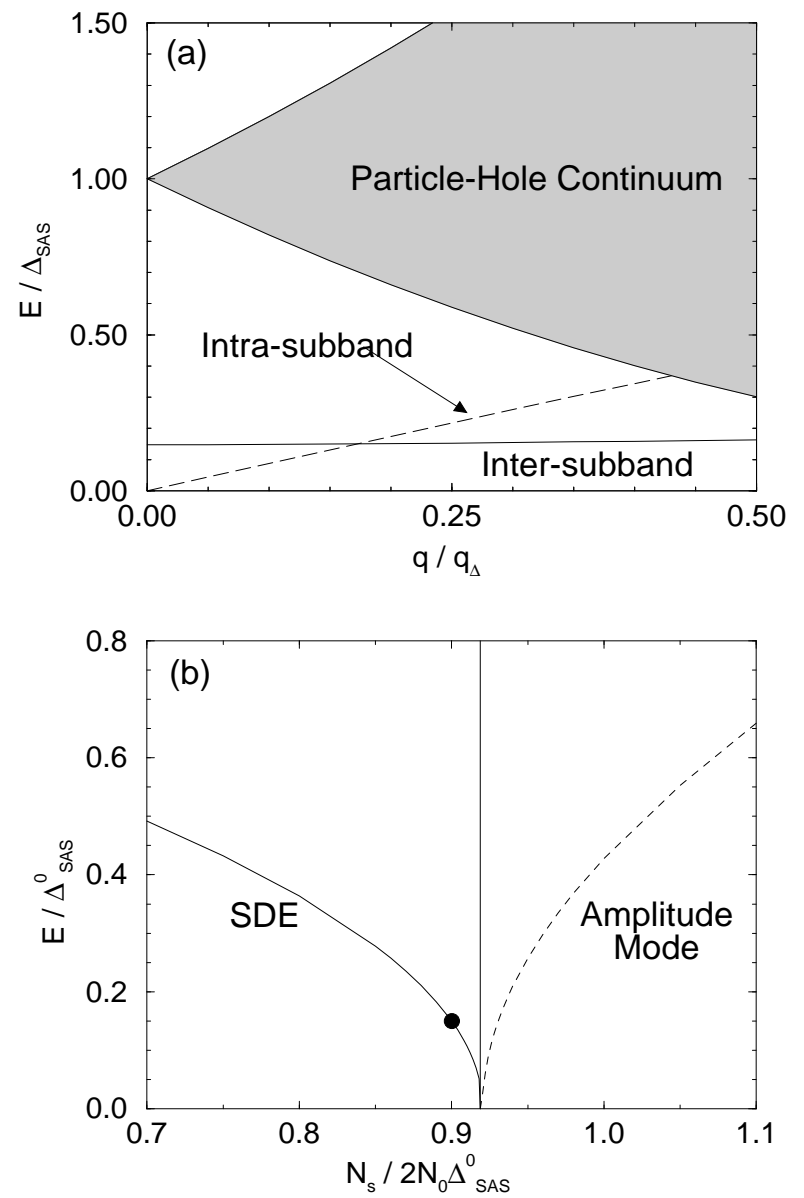

FIG. 5. Collective spin-density excitations in a symmetric double quantum well of the type shown in Fig. 2 with $d U=0$. (a) Collective mode spectrum in terms of excitation energy $E$ and intra-well wave vector $q$ showing the inter- (solid line) and intra- (dashed line) collective spin-density excitations as well as the continuum of inter-subband particle-hole excitations (shaded area). Although not apparent in the figure, the inter-subband collective mode does disperse with $q$. (b) $q=0$ inter-subband spin density excitation (SDE) energy $E$ as the sheet density $N_{s}$ is tuned through the $N_{1}$ to $A F$ transition at fixed interaction strength $N_{0} V_{12}=0.55$ [cf. Fig. 4 (a)]. Note that the SDE (solid line) softens completely at the transition point and that the collective mode appearing on the antiferromagnetic side $\left(N_{s} / 2 N_{0} \Delta_{\text {SAS }}^{0}>0.919\right)$ is the amplitude mode of the antiferromagnetic order parameter (dashed line). The dot indicates the point in parameter space presented in (a). In this figure, $N_{0}$ is the single-spin, $2 \mathrm{D}$ electronic density of states, $\Delta_{\text {SAS }}^{0}$ is the energy separation of the lowest two subbands in a non-interacting double quantum well, $\Delta_{\text {SAS }}$ is the splitting in the interacting system, and $q_{\Delta}^{2}=2 m^{*} \Delta_{\text {SAS }}^{0} / \hbar^{2}$. The intra-band particle-hole continuum is not indicated. turns into the collective mode associated with amplitude fluctuations of the antiferromagnetic order parameter.15 Experimentally, then, one expects to see a complete softening of the inter-subband SDE and the recovery of this amplitude mode as the density is tuned through the transition.

The presence of asymmetry in the double quantum well complicates this picture somewhat. The SDE spectrum for our asymmetric $(d U=0.5 \mathrm{meV})$ double quantum well illustrates these complications and is shown in Fig. 6(a). Most noticeably, the asymmetry mixes the intra- and inter-subband excitations, so that even in scattering geometries designed to measure only inter-subband response both intra- and inter-subband excitations will appear.27 This effect is seen through both an enlarged particle-hole continuum and the presence of a damped mode in the inter-subband spectrum corresponding to the intra-subband SDE. Furthermore, the asymmetry couples the intra- and inter-subband SDEs themselves, leading to an avoided crossing which may be seen by comparing Fig. 6(a) to Fig. 5(a).

This avoided crossing effectively prevents the intersubband SDE from completely softening on entering the antiferromagnetic phase, as shown in Fig. E(b). In this figure, ones sees that, as the density is tuned toward the antiferromagnetic transition, the $q=0$ inter-subband SDE energy decreases to a finite value. Further increase of the density into the antiferromagnetic phase reverses this trend, and the energy of the amplitude mode of the antiferromagnetic order parameter increases, resulting in a cusp.

Despite the fact that the inter-subband SDE does not completely soften, the antiferromagnetic phase appears, demonstrating that this softening is a sufficient but not necessary signature of the zero-field phase transition. We expect some mode to soften at this transition, however, and it turns out that it is the intra-subband SDE which collapses. Specifically, the mode coupling between interand intra-subband SDEs pushes the latter mode down in energy at all $q$, effectively reducing the group velocity of this mode. Approaching the antiferromagnetic transition, the $q \rightarrow 0$ group velocity of the intra-subband SDE decreases until it vanishes at the transition point. The resulting phase has the character of the real-space spin density profile of the intra-subband mode, which direct calculation reveals to be antiferromagnetic. The character of the intra-subband excitation at small $q$ is therefore very similar to the inter-subband excitation in the symmetric well due to the mode coupling between the two excitations. Thus, its softening can lead to an antiferromagnetic transition without inconsistency.

\section{CONCLUSION}

In this paper, we have examined the origin and robustness of a zero-field antiferromagnetic phase in dou- 

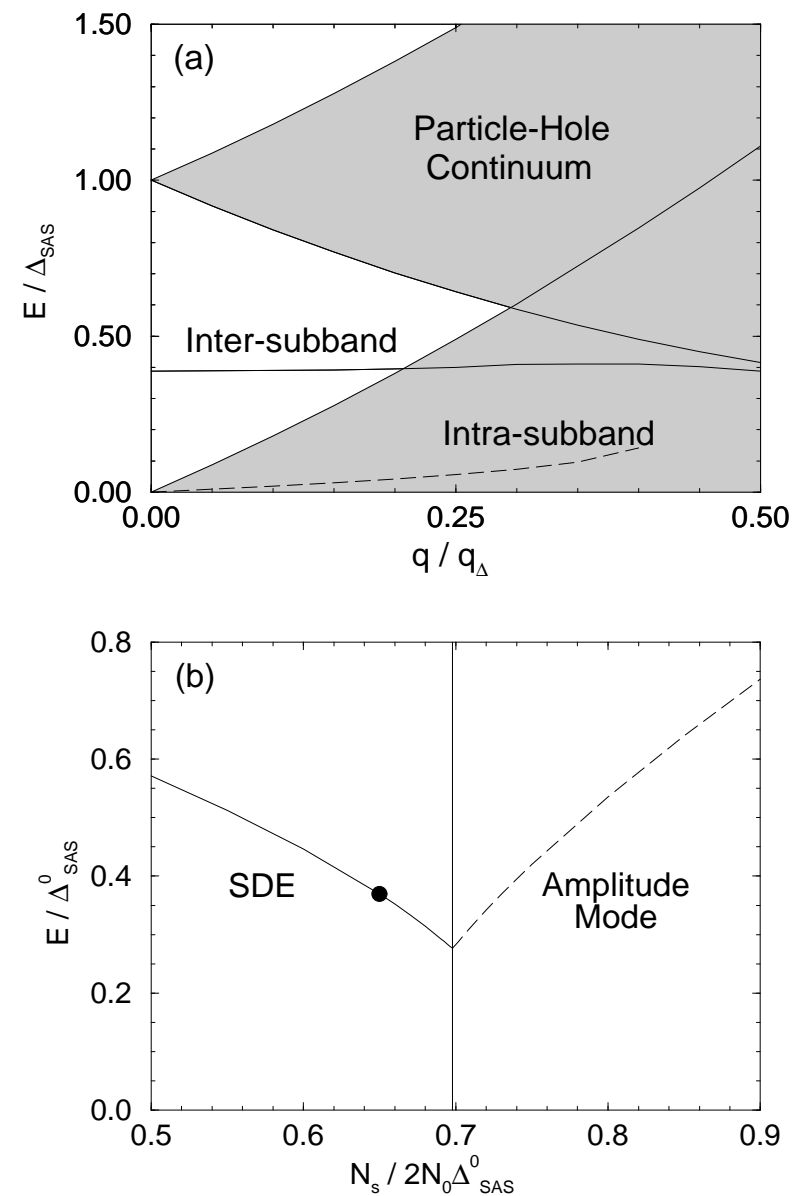

FIG. 6. Collective spin-density excitations in a weakly asymmetric double quantum well of the type shown in Fig. 2 with $d U=0.5 \mathrm{meV}$. The organization of and notation in the figure are the same as Fig. 0 with the interaction strength fixed at $N_{0} V_{12}=0.55$ and the antiferromagnetic transition occurring at $N_{s} / 2 N_{0} \Delta_{\text {SAS }}^{0}=0.698$. As seen in (a), the asymmetry induces a coupling between the inter- and intra-subband spin-density excitations which results in the avoided crossing of inter- and intra-subband spin-density dispersions and a mixing of intra-subband particle-hole excitations with the inter-subband continuum. In (b), one sees that this mode coupling also prevents the inter-subband SDE from softening when going from the paramagnetic to the antiferromagnetic phase. ble quantum wells predicted in previous calculations 14,15 Based on a simple model, we determined that such magnetic phases are a direct consequence of the magnetism expected at low densities in two-dimensional systems where interaction effects dominate. In particular, magnetic phases with either ferro- or antiferromagnetic spin polarizations are possible, and a superexchange interaction between the wells leads to a region at intermediate densities and interaction strengths where the antiferromagnetic phase is preferred.

By performing a detailed self-consistent Hartree-Fock calculation on a model of these double quantum well systems, we addressed two features left out of preceding work:15 intra-subband repulsion and asymmetry of the heterostructure. Our results support and extend those of Ref. 15. Specifically, we found that the antiferromagnetic phase is stable in a large region of the model phase diagram despite the presence of intra-subband repulsion that is as strong as the inter-subband repulsion which drives the antiferromagnetic instability. The intrasubband repulsion does, however, introduce ferromagnetic phases, producing a rich phase diagram. Note, however, that no charge ordering phases of the type discussed in Refs. 210 were observed, even though our formalism did allow for that possibility. In addition, both ferroand antiferromagnetic phases persist in the presence of asymmetry in the quantum well structure; indeed, the phase diagram is qualitatively unaffected by its introduction. Asymmetry does have a strong influence on the collective mode spectrum, though, and induces a modecoupling between inter- and intra-subband spin-density excitations which prevents the latter from completely softening at the antiferromagnetic phase transition. Instead, the inter-subband spin-density excitation exhibits a cusp at the transition while the $q \rightarrow 0$ group velocity of the intra-subband excitation vanishes. The coupling between these two modes nonetheless lends an antiferromagnetic character to the intra-subband excitation in the asymmetric system and enables the collapse of its group velocity to yield the antiferromagnetic phase.

Taken together, these results strongly indicate that, if an exchange-correlation-induced ferromagnetic transition occurs in a single-layer system at sufficiently low density, then the antiferromagnetic phase in a doublelayer system should also occur. However, the issue of whether or not the ferromagnetic phase obtains in a single two-dimensional layer is not settled. Variational Monte Carlo calculations 28,29 show the presence of a ferromagnetic phase between the paramagnetic and Wigner crystal phases, but Green's function Monte Carlo computations 29 find no intervening ferromagnetism. More recent numerical work based on the Monte Carlo technique $30,31,8$ once again favors the existence of a ferromagnetic transition. Other calculations using density-fupctional theory in the local-spin-density approximation 1510 also support the existence of a ferromagnetic transition at sufficiently low density. Thus, although a definitive demonstration of ferromagnetism in 
a single, two-dimensional layer is lacking, a large body of evidence exists which firmly supports this hypothesis.

The precise conditions under which these phases would be observable are somewhat harder to elucidate based on the mean-field theory presented in this paper. The principle difficulty is that mean-field calculations will tend to overestimate the temperatures and densities at which exchange-correlation-induced phase transitions occur. The source of this difficulty lies in the neglect of fluctuations in the theory, which play an important role in the low-dimensional structures considered. If one goes to densities and temperatures deep below the critical values of these parameters, we expect that the mean-field thepry will give an accurate qualitative picture of the phase. 32

In sum, work on the single layer system indicates that its ferromagnetic state is unlikely to occur for electrongas density parameters smaller than $r_{s} \approx 10$, a much lower density than would be indicated by the HartreeFock approximation, for which the transition to the ferromagnetic state occurs at $r_{s} \approx 2$. The present work suggests that the two-layer antiferromagnetic phase, as well as two-layer ferromagnetic phases, are likely to be present in double-layer systems when the density per layer approaches the low value at which the single-layer ferromagnetic instability occurs. For the GaAs systems studied experimentally it therefore seems unlikely that the antiferromagnetic state will occur for densities per layer substantially larger than $\approx 10^{10} \mathrm{~cm}^{-2}$. However, it is exceedingly difficult to estimate the transition density theoretically and one must rely on experiment.

Currently, a single experimental publication regarding a search for the antiferromagnetic phase has appeared in the literature, 18 and the results are equivocal. The authors of this study report inelastic light scattering measurements of the long-wavelength inter-subband collective spin-density excitations as a function of density in a double quantum well which was expected, on the basis of the original theoretical work, to show the antiferromagnetic instability 18 Instead of completely staning at a finite density, as predicted in earlier work, 245 the intersubband SDE shows no dramatic structure down to the lowest densities measured 18 These results could be accounted for in at least two different ways. The most likely explanation is that the electron density of the sample, of order $10^{11} / \mathrm{cm}^{2}, 18$ is above the critical density for the antiferromagnetic transition. Alternatively, the calculations in this paper demonstrate that slight asymmetry in the double quantum well will prevent a complete softening of the inter-subband SDE and yield a cusp as a function of density. Since the energy of the amplitude mode in the antiferromagnetic phase is similar to that of the inter-subband SDE away from the critical density [cf. Fig. $6(\mathrm{~b})]$, measurements at a closely spaced grid of densities may be required to detect this cusp. In addition, the cusp may be broadened by impurity or fluctuation effects which are beyond our mean-field theory, further increasing the difficulty of detecting the transition. Thus, the current experimental results cannot exclude the ex- istence of the zero-field antiferromagnetic state, and its robustness as demonstrated by the calculations in this paper leaves us optimistic that such a unique exchangedriven phase can occur in nature.

\section{ACKNOWLEDGMENTS}

RJR would like to acknowledge stimulating discussions with $\mathrm{H}$. Ehrenreich regarding the superexchange mechanism in this system. The work of RJR and SDS work was supported by the US-ARO and the US-ONR. The work of AHM was supported by the National Science Foundation under grant DMR-9416906.

${ }^{1}$ For a recent review, see Perspectives in Quantum Hall Effects, edited by S. Das Sarma and A. Pinczuk (Wiley, New York, 1997).

${ }^{2}$ A. H. MacDonald, Phys. Rev. B 37, 4792 (1988).

${ }^{3}$ P. P. Ruden and Z. Wu, Appl. Phys. Lett. 59, 2165 (1991).

${ }^{4}$ L. Świerkowski, D. Neilson, and J. Szymański, Phys. Rev. Lett. 67, 240 (1991); D. Neilson, L. Świerkowski, J. Szymański, and L. Liu, ibid. 71, 4035 (1993).

${ }^{5}$ C. M. Varma, A. I. Larkin, and E. Abrahams, Phys. Rev. B 49, 13999 (1994).

${ }^{6}$ Y. Katayama, D. C. Tsui, H. C. Manoharan, and M. Shayegan, Surf. Sci. 305, 405 (1994); Phys. Rev. B 52, 14817 (1995).

${ }^{7}$ X. Ying, S. R. Parihar, H. C. Manoharan, and M. Shayegan, Phys. Rev. B 52, 11611 (1995).

${ }^{8}$ N. K. Patel, I. S. Millard, E. H. Linfield, P. D. Rose, M. P. Grimshaw, D. A. Ritchie, G. A. C. Jones, and M. Pepper, Phys. Rev. B 53, 15443 (1996).

${ }^{9}$ S. Conti and G. Senatore, Europhys. Lett. 36, 695 (1996).

${ }^{10}$ L. Zheng, M. W. Ortalano, and S. Das Sarma, Phys. Rev. B 55, 4506 (1997).

${ }^{11}$ X.G. Wen and A. Zee, Phys. Rev. Lett. 69, 1811 (1992); X.G. Wen and A. Zee, Phys. Rev. B 47, 2265 (1993).

12 Z.F. Ezawa and A. Iwazaki, Int. J. Mod. Phys. B 6, 3205 (1992); ibid., 8, 2111 (1994); Phys. Rev. B 47, 7295 (1993); 48, 15189 (1993); Phys. Rev. Lett. 70, 3119 (1993); Z.F. Ezawa, A. Iwazaki, and Y.S. Wu, Mod. Phys. Lett. B 7, 1223 (1993); 7, 1825 (1993); Z.F. Ezawa, Phys. Rev. B, Phys. Rev. B 51, 11152 (1995).

${ }^{13}$ Kun Yang, K. Moon, L. Zheng, A.H. MacDonald, S.M. Girvin, D. Yoshioka, and Shou-Cheng Zhang, Phys. Rev. Lett. 72, 732 (1994); K. Moon, H. Mori, Kun Yang, S. M. Girvin, A. H. MacDonald, L. Zheng, D. Yoshioka, and Shou-Cheng Zhang, Phys. Rev. B, 51, 5138 (1995); K. Moon, H. Mori, Kun Yang, S.M. Girvin, A.H. MacDonald, L. Zheng, D. Yoshioka, and Shou-Cheng Zhang, submitted to Phys. Rev. B (1995).

${ }^{14}$ S. Das Sarma and P. I. Tamborenea, Phys. Rev. Lett. 73, 1971 (1994). 
${ }^{15}$ R. J. Radtke, P. I. Tamborenea, and S. Das Sarma, Phys. Rev. B 54, 13832 (1996).

${ }^{16}$ V. Pellegrini, A. Pinczuk, B. S. Dennis, A. S. Plaut, L. N. Pfeiffer, and K. W. West, Phys. Rev. Lett. 78, 310 (1997).

17 L. Zheng, R. J. Radtke, and S. Das Sarma, Phys. Rev. Lett. 78, 2453 (1997).

18 A. S. Plaut, A. Pinczuk, P. I. Tamborenea, B. S. Dennis, L. N. Pfeiffer, and K. W. West, Phys. Rev. B 55, 9282 (1997).

${ }^{19}$ See, for example, S. Doniach and E. H. Sondheimer, Green's Functions for Solid State Physicists (Addison-Wesley, New York, 1982), Chap. 7.

${ }^{20}$ L. Swierkowski and A.H. MacDonald, Phys. Rev. B 55, R16017 (1997).

${ }^{21}$ G. D. Mahan, Many-Particle Physics (Plenum, New York, 1981).

22 A. Pinczuk and G. Abstreiter, in Light Scattering in Solids $V$, edited by M. Cardona and G. Guntherodt (SpringerVerlag, Berlin, 1989).

${ }^{23}$ S. Das Sarma, in Light Scattering in Semiconductor Structures and Superlattices, edited by D. J. Lockwood and J. F. Young (Plenum Press, New York, 1991).

${ }^{24}$ A.H. MacDonald, P.M. Platzman and G.S. Boebinger, Phys. Rev. Lett. 65, 775 (1990).

25 T. Ando, A. B. Fowler, and F. Stern, Rev. Mod. Phys. 54, 437 (1981).

${ }^{26}$ A. Pinczuk, S. Schmitt-Rink, G. Danan, J. P. Valladares, L. N. Pfeiffer, and K. W. West, Phys. Rev. Lett. 63, 1633 (1989).

${ }^{27}$ We shall continue to refer to the SDEs as inter- and intra-subband in an asymmetric double quantum well even though that distinction does not strictly apply. The reader should understand these terms to refer to the dominant character of the collective excitation with respect to the symmetric structure.

${ }^{28}$ D. M. Ceperley and B. J. Alder, Phys. Rev. Lett. 45, 566 (1980); J. Perdew and A. Zunger, Phys. Rev. B 23, 5048 (1981).

29 B. Tanatar and D. M. Ceperley, Phys. Rev. B 39, 5005 (1989).

${ }^{30}$ D. M. Ceperley, private communication.

${ }^{31}$ F. Rapisarda and G. Senatore, Aust. J. Phys. 49, 161 (1996).

32 Technically, the Mermin-Wagner theorem [N. D. Mermin and H. Wagner, Phys. Rev. Lett. 17, 1133 (1966)] implies that fluctuations will completely destroy long-range order in two-dimensional systems of infinite extent at nonzero temperature. In practice, however, the finite sample size prohibits arbitrarily long-wavelength fluctuations and yields a region of finite temperature in which the sample is effectively a single, ordered domain. It is in this region where the mean-field theory should yield a good description of the antiferromagnetic phase. 